DOI: $10.1590 / 1089-6891 v 17 i 321194$

PRODUÇÃO ANIMAL

\title{
CONFORMAÇÃO CORPORAL DE EQUINOS DE DIFERENTES GRUPOS GENÉTICOS
}

\author{
CONFORMATION INDEX OF HORSES OF DIFFERENT GENETIC GROUPS
}

\author{
Marcos Paulo Gonçalves Rezende ${ }^{1^{*}}$ \\ Julio Cesar de Souza² \\ Marcelo Falci Mota ${ }^{3}$ \\ Nicacia Monteiro Oliveira ${ }^{2}$ \\ Rodrigo José Delgado Jardim ${ }^{4}$
}

\author{
${ }^{1}$ Universidade Estadual do Sudoeste da Bahia, Itapetinga, BA, Brasil. \\ ¿Universidade Federal do Mato Grosso do Sul, Campo Grande, MS, Brasil. \\ 3Universidade Federal da Fronteira do Sul, Chapecó, SC, Brasil. \\ ${ }^{4}$ Universidade Federal Rural de Pernambuco, Recife, PE, Brasil. \\ *Autor para correspondência - mpgrezende@gmail.com
}

\begin{abstract}
Resumo
Qualquer atividade equestre exig e equino com aptidão adequada, podendo ser identificada através da conformação corporal (IC), calculada pela combinação de medidas biométricas. Objetivouse avaliar IC de 86 equinos, pertencentes aos grupos genéticos (GG): Crioulo (CR), Brasileiro de Hipismo (BH), Árabe (ARB), Quarto de Milha (QM), Puro Sangue Inglês (PSI) e mestiços com pequena estatura corporal, rotulado Petiço (PE). Avaliaram-se relação cernelha garupa, peso corporal estimado, torácico, corporal, corporal relativo, conformação, compacidade e carga 1 e 2, carga na canela e dáctilo-torácico. Analisaram-se efeito de sexo $(\mathrm{S}), \mathrm{GG}$ e interação $\mathrm{S}^{*} \mathrm{GG}$; correlações entre IC; componentes principais, elaborando-se diagrama de dispersão com biplot, para se verificar a distribuição dos GG em relação aos IC; e cluster para verificar distância entre GG. Os efeitos foram responsáveis por variações $(\mathrm{P}<0,05)$ nos IC. Considerou-se o $\mathrm{PE}$ elipométrico e os demais, eumétricos. Considerou-se o PSI mediolíneo e os demais, brevilíneos. Equinos PSI, QM e PE apresentaramse aptos para executar atividades com sela até tração leve e o restante, até tração pesada. Equinos BH e ARB apresentaram maior capacidade de suportar peso sem esforço exagerado sobre o dorso, trabalhando a passo e ao galope. Observaram-se maior similaridade entre QM e CR e menor entre PE com os GG.
\end{abstract}

Palavras-chave: aptidões; diversidade; equinos; conformação.

\begin{abstract}
Any equestrian activity requires horses with proper fitness, which can be identified through body conformation (BC), calculated by combined biometric measurements. The objective of this study was to evaluate the $\mathrm{BC}$ of 86 horses belonging to genetic groups (GG): Crioulo (CR), Brazilian Equestrian (BE), Arabian (ARB), Quarter Horse (QH), Thoroughbred (THO), and a crossbred group with small stature, named as Petiço (PE). We evaluated the relationship between shoulder and withers, estimated body weight, length, weight, weight relative, conformation, compactness and load 1 and 2, load on leg and dactyl-thoracic. The variation sources in the model were the effects of gender, GG, and the interaction $\mathrm{GG}^{*}$ gender; the correlation between $\mathrm{BC}$; principal components analysis, preparing a scatter plot with biplot to verify the distribution of GG in relation to the $\mathrm{BC}$; cluster to check distance
\end{abstract}


between GG. The variation sources affected $\mathrm{BC}(\mathrm{P}<0.05)$. PE was considered elipometric, while the others were eumetric. THO was considered of medium shape, and the others as having small shape. Horses THO, QH, PE were qualified for activities with saddle up to light traction, and the rest of horses from saddle to heavy traction. Horses BE and ARB had greater capacity to support weight without overexertion on the back, working step and gallop. There were greater similarities between QM and CR, and lower among PE and the other GGs.

Keywords: diversity; equines; phenotypic; skills.

Enviado em: 26 novembro de 2011

Aceito em: 25 abril de 2016.

\section{Introdução}

A subdivisão ou estruturação em espécies de animais domésticos é, geralmente, mais evidente do que em espécies selvagens, devido à separação após serem diferenciadas raças e/ou grupo genéticos geneticamente superiores para uso local, regional ou nacional ${ }^{(1)}$. Assim, compreender as diferenciações fenotípicas é fundamental para identificar as aptidões específicas entre os grupos genéticos, tendo em vista que, para praticar qualquer tipo de atividade equestre, são necessários equinos com perfil corporal compatível ${ }^{(2,3)}$.

Para tal fim, os índices de conformação corporal constituem ferramentas interessantes para selecionar e proceder à caracterização e diferenciação racial, estimadas por meio das associações entre medidas lineares de várias partes do corpo do animal ${ }^{(3-9)}$. Adicionalmente, são fáceis mensurações e não oscilam dentro de determinado período ${ }^{(10)}$.

De acordo com Rezende et al..$^{(9)}$, Martin-Rosset ${ }^{(11)}$ e Franci et al. ${ }^{(12)}$, por meio do conhecimento de índices de conformação corporal, podem-se obter informações sobre as aptidões dos equinos, como a verificação do equilíbrio entre membros locomotores do animal, do tipo (hipermétricos, eumétricos e elipométricos), da característica (longilíneo, mediolíneo e brevilíneo), da aptidão (sela, tração e/ou dupla aptidão), e da capacidade corporal (peso sem esforço exagerado que o animal pode suportar sobre o dorso, trabalhando a trote ou a galope ou trabalhando a passo). Assim, objetivou-se analisar a conformação corporal de seis grupos genéticos de equinos.

\section{Material e Métodos}

$\mathrm{O}$ estudo foi conduzido em parcerias com diferentes centros equestres de cavalos no município de Campo Grande (Haras do Zharan, Jockey Clube Municipal e Centro de Hipismo do Exército Brasileiro), no município de Miranda (Fazenda Bodoquena) e município de Aquidauana (Fazenda Taboco), estado do Mato Grosso do Sul, Brasil, durante os meses de fevereiro a junho de 2011.

Foram utilizados 86 equinos (Tabela 1), com idade adulta, pertencentes aos grupos genéticos Crioulo (CR), Brasileiro de Hipismo (BH), Árabe (ARB), Quarto de Milha (QM), Puro Sangue Inglês (PSI); e um grupo formado de equinos com pequena estatura corporal e sem raça definida, rotulado como grupo genético Petiço (PE), considerado um equídeo pequeno e não um pônei, em virtude de suas características fenotípicas ${ }^{(13)}$. A inclusão do grupo genético Petiço no estudo, justifica-se pelo seu uso no estado do Mato Grosso do Sul, como animais para corridas de curtas distâncias e no manejo de bovinos. 
Tabela 1. Descrição quantitativa e qualitativa dos grupos genéticos de equinos criados em diferentes criatórios no Mato Grosso do Sul

\begin{tabular}{ccccc}
\hline Grupo & \multicolumn{2}{c}{ N } & & Frequência \\
\cline { 2 - 5 } & Fêmeas & Machos & Total & Fr (\%) \\
\hline ARB & 14 & 6 & 20 & 23,15 \\
PSI & 5 & 9 & 14 & 16,27 \\
CR & 7 & 11 & 18 & 20,93 \\
PE & 4 & 3 & 7 & 8,13 \\
BH & 7 & 8 & 15 & 17,44 \\
QM & 8 & 4 & 12 & 13,95 \\
\hline Total & 45 & 41 & 86 & $100 \%$ \\
\hline
\end{tabular}

ARB: Árabe; PSI: Puro Sangue Inglês; CR: Crioulo; BH: Brasileiro de Hipismo; QM: Quarto de Milha; PE: grupamento genético Petiço. N: quantidade de animais; Fr (\%): Frequência relativa.

Conforme Rezende et al. ${ }^{(4)}$, Parés Casanova ${ }^{(7)}$, Rezende et al..$^{(8)}$, Oom e Ferreira ${ }^{(14)}$ e Peña et al. ${ }^{(15)}$, as mensurações foram realizadas sempre do lado esquerdo do corpo do equino, posicionando-o com a menor irregularidade possível em relação ao solo, medindo-o com auxílio de fita métrica e hipômetro. As seguintes medidas foram aferidas: perímetro torácico, altura de cernelha, comprimento corporal, largura de peito e largura de canela. Com base nos valores determinados nas medidas e de acordo com as metodologias descritas na literatura ${ }^{(8,9,11,12)}$, estimaram-se 12 índices de conformação:

1. Índice de relação cernelha e garupa (RCG): altura de cernelha dividida pela altura de garupa. Valor igual a 1, descreve animal com membros torácicos e pélvicos de mesmas alturas (equilíbrio).

2. Índice dáctilo-torácico (IDT): perímetro de canela dividido pelo perímetro torácico. Animais hipermétricos apresentam IDT $>11,5$, eumétricos 10,5 $\leq$ IDT $\leq 10,8$ e hipométricos IDT $<10,5$.

3. Índice de peso corporal estimado $(\mathrm{P})$ : perímetro torácico elevado ao cubo multiplicado pela constante. Animais hipermétricos apresentam $\mathrm{P}>550 \mathrm{~kg}$, eumétricos $350 \leq \mathrm{P} \leq 550 \mathrm{~kg}$ e elipométrico $\mathrm{P}<350 \mathrm{~kg}$.

4. Índice corporal (IC): comprimento do corpo dividido pelo perímetro torácico. Animais longilíneos apresentam IC $>0,90$, mediolíneos $0,86 \leq \mathrm{IC} \leq 0,88$ e animais brevilíneos $\mathrm{IC}<0,85$.

5. Índice torácico (IT): largura do peito dividida pelo perímetro torácico. Animais longilíneos apresentam IT $<0,85$, mediolíneos $0,86 \leq \mathrm{IC} \leq 0,88$ e animais brevilíneos $\mathrm{IC}>0,85$.

6. Índice de conformação (ICF): perímetro torácico elevado ao quadrado dividido pela altura da cernelha. Animais de sela apresentam ICF $\leq 2,1125$ e tração ICF $>2,1125$.

7. Índice de carga 1 (ICG1): perímetro torácico elevado ao quadrado e multiplicado pela constante 56, dividido pela altura da cernelha.

8. Índice de carga 2 (ICG2): perímetro torácico elevado ao quadrado e multiplicado pela constante 95 , dividido pala altura da cernelha.

9. Índice corporal relativo (ICR): comprimento do corpo multiplicado pela constante 100 e dividido pela altura da cernelha.

10. Índice de compacidade 1 (ICO1): peso estimado dividido pela altura da cernelha, dividindo-se esta relação por 100 . Animais de tração pesada apresentam ICO1 > 3,15, tração ligeira ICO1 $\approx 2,75$ e sela $\approx 2,60$.

11. Índice de compacidade 2 (ICO2): peso estimado dividido pela altura da cernelha subtraída do valor 1 e dividindo-se esta relação por 100. Animais de tração pesada apresentam ICO2 >9,5, animais 
de tração leve $8,0 \leq \mathrm{ICO} 2 \leq 9,5$ e sela $6,0 \leq \mathrm{ICO} 2 \leq 7,75$.

12. Índice de carga na canela (ICC): perímetro da canela ao cubo, dividido pelo peso corporal, sendo o resultado multiplicado por 100.

Para se verificar a existência de efeito de sexo, grupo genético e interação sexo e grupo genético sobre os índices de conformação, realizou-se análise de variância utilizando-se o método dos quadrados mínimos, com o seguinte modelo estatístico:

$$
Y_{i j}=m+S_{i}+G_{j}+G S i j+E_{i j k}
$$

Em que: Yij = variáveis dependentes; $\mathrm{m}=$ média de todas as observações; $\mathrm{Si}=$ efeito de sexo de ordem $\mathrm{i} ; \mathrm{Gj}=$ grupo genético de ordem $\mathrm{j} ; \mathrm{GSij}$ = interação grupo genético*sexo; Eijk = erro aleatório. As médias e erros padrões foram liberados na análise de variância. Posteriormente, realizaram-se análises de correlação de Pearson entre os índices de conformação. Utilizando-se o Software Past versão 2.17 , realizou-se análise de componentes principais a partir da matriz de correlação ${ }^{(16)}$ dos valores médios dos grupos genéticos. O critério adotado para selecionar os componentes principais foi com base no valor mínimo de $70 \%$ da variância acumulada.

Realizou-se um diagrama de dispersão com biplot, para se verificar a distribuição dos seis grupos genéticos em relação aos componentes principais. Para se verificar a dissimilaridade entre os grupos genéticos, realizou-se por meio da análise de cluster um dendrograma por algoritmo de grupo emparelhado considerando-se a medida de similaridade euclidiana; e para avaliar a consistência no processo de agrupamento, utilizou-se o coeficiente de correlação cofenética proposto por Skoal e Rohlf(17).

\section{Resultados}

O grupo genético, o sexo e a interação grupo genético*sexo foram responsáveis por variações $(\mathrm{P}<0,05)$ nos índices de conformação entre os equinos (Tabela 2), e os maiores coeficientes de variações foram encontrados para os ICO2, P e ICO1, e os menores para os RCG, ICR e IC.

Tabela 2. Resumo da análise de variância (ANOVA) dos índices de conformação entre os equinos

\begin{tabular}{|c|c|c|c|c|c|c|c|c|c|c|c|c|}
\hline & RCG & IDT & $\mathbf{P}$ & IC & IT & ICF & ICGl & ICG2 & ICR & ICOI & ICO2 & ICC \\
\hline $\mathrm{G}$. & $* * *$ & $* * *$ & $* * *$ & **** & $* * *$ & $* * *$ & $* * *$ & $* * *$ & $* * *$ & $* * *$ & ***** & $* * *$ \\
\hline S. & * & $* * *$ & ns & ns & $* *$ & $\mathrm{~ns}$ & ns & ns & $* * *$ & ns & $* * *$ & ns \\
\hline G*S & ns & $* * *$ & $* * *$ & $*$ & ns & $* * * *$ & $* * *$ & $* * *$ & $* *$ & $* * *$ & $* * *$ & $* * *$ \\
\hline $\mathrm{M}$ & 1,00 & 0,10 & 464,58 & 0,84 & 0,21 & 2,13 & 119,35 & 202,47 & 99,89 & 3,06 & 9,47 & 0,04 \\
\hline CV\% & 1,40 & 5,77 & 10,57 & 4,28 & 8,36 & 6,08 & 6,08 & 6,08 & 3,51 & 9,43 & 14,17 & 9,43 \\
\hline
\end{tabular}

Os equinos BH e ARB apresentaram maior equilíbrio entre os membros locomotores (Tabela 3). Considerou-se o grupo PE elipométrico e os demais, eumétricos. O grupo PSI demonstrou característica mediolínea, enquanto que o BH, ARB, QM, PE e CR, brevilínea. Considerou-se o PSI, QM, PE aptos para atividades com sela até tração leve e os demais habilitados ao uso de sela e ao trabalho de tração pesada. O BH e ARB apresentaram maior capacidade de suportar peso sem esforço exagerado sobre o dorso, trabalhando a passo e ao galope em relação aos demais. $\mathrm{O}$ grupo $\mathrm{PE}$ apresentou maior comprimento corporal em relação à sua altura, enquanto que esta proporcionalidade no grupo CR foi igual e, para os demais grupos, observou-se maior altura em relação ao comprimento corporal. O PE apresentou maior capacidade de suporte de peso sobre os membros locomotores. 
Tabela 3. Média e erro padrão dos índices de conformação dos diferentes genótipos de equinos com base no teste de Tukey a $5 \%$ de probabilidade

\begin{tabular}{|c|c|c|c|c|c|c|}
\hline Grupo & RCG & IDT & $\mathbf{P}$ & IC & IT & ICF \\
\hline PSI & $\begin{array}{l}0,99 \pm \\
0,00 \mathrm{e}^{* *}\end{array}$ & $\begin{array}{l}0,10 \pm \\
0,00 \text { bc }^{8}, \mathrm{e}^{* 8}\end{array}$ & $\begin{array}{l}484 \pm \\
14^{b^{*}, \mathrm{ce}^{* *}, \mathrm{f}^{* * *}}\end{array}$ & $\begin{array}{l}0,87 \pm \\
0,01 \mathrm{e}^{* *,}, \mathrm{~d}^{* * 8}\end{array}$ & $\begin{array}{l}0,21 \pm \\
0,00 \text { be }^{* *}\end{array}$ & $\begin{array}{l}2,08 \pm \\
0,03 \mathrm{~d}^{*}\end{array}$ \\
\hline $\mathrm{BH}$ & $\begin{array}{l}1,00 \pm \\
0,00 \mathrm{f}^{*}\end{array}$ & $\begin{array}{l}0,11 \pm \\
0,00 \mathrm{a}^{*} \mathrm{~d}^{* *}\end{array}$ & $\begin{array}{l}527 \pm \\
13^{\mathrm{a}^{*}, \mathrm{~d}^{*}, \text {, cef }} * *\end{array}$ & $\begin{array}{l}0,85 \pm \\
0,00 \mathrm{e}^{* *,}, \mathrm{~d}^{* * 8}\end{array}$ & $\begin{array}{l}0,23 \pm \\
0,00 \mathrm{a}^{*}, \mathrm{dff}^{* 8}, \mathrm{e}^{* * *}\end{array}$ & $\begin{array}{l}2,17 \pm \\
0,03 \mathrm{f}^{*} \mathrm{c}^{\mathrm{c} *}\end{array}$ \\
\hline $\mathrm{QM}$ & $\begin{array}{l}0,99 \pm \\
0,00 \mathrm{~d}^{*}, \mathrm{e}^{\mathrm{e} *}\end{array}$ & $\begin{array}{l}0,11 \pm \\
0,00^{a^{*}, d^{2 *}}\end{array}$ & $\begin{array}{l}424 \pm \\
15,04 \text { adf }^{* *}, b^{* * *}\end{array}$ & $\begin{array}{l}0,85 \pm \\
0,01 \mathrm{e}^{* ;}, \mathrm{d}^{* *}\end{array}$ & $\begin{array}{l}0,22 \pm \\
0,00 \mathrm{f}^{*}, \mathrm{e}^{* *}\end{array}$ & $\begin{array}{l}1,99 \pm \\
0,03 \text { bde }^{* *}\end{array}$ \\
\hline $\mathrm{ARB}$ & $\begin{array}{l}1,00 \pm \\
0,00^{\mathrm{c}^{*}, \mathrm{f}^{* *}}\end{array}$ & $\begin{array}{l}0,10 \pm \\
0,00^{f^{*}, b c^{*}, e^{* * *}}\end{array}$ & $\begin{array}{l}483 \pm \\
11,99 \text { bce**,f** }\end{array}$ & $\begin{array}{l}0,81 \pm \\
0,00 \\
\mathrm{f}^{*}, b \mathrm{c}^{* 8}, \mathrm{a}^{* * *}\end{array}$ & $\begin{array}{l}0,21 \pm \\
0,00^{b^{*}}\end{array}$ & $\begin{array}{l}2,18 \pm \\
0,03 \mathrm{a}^{*}, \mathrm{fc} * *\end{array}$ \\
\hline $\mathrm{CR}$ & $\begin{array}{l}1,01 \pm \\
0,00 \text { acf }^{* * *}\end{array}$ & $\begin{array}{l}0,11 \pm \\
0,00 \mathrm{a}^{\mathrm{a}^{* 8}, \mathrm{~d}^{* * 8}}\end{array}$ & $\begin{array}{l}432,26 \pm \\
12^{\text {ad }^{* *}, \mathrm{bf}^{* * 8}}\end{array}$ & $\begin{array}{l}0,82 \pm \\
0,00 \mathrm{ac}^{*}, b^{* 8}\end{array}$ & $\begin{array}{l}0,20 \pm \\
0,00 \mathrm{a}^{*}, \mathrm{c}^{* *}, \mathrm{~b}^{* * *}\end{array}$ & $\begin{array}{l}2,12 \pm \\
0,03 \mathrm{c}^{* 8}\end{array}$ \\
\hline $\mathrm{PE}$ & $\frac{0,98 \pm}{0,00^{b^{*}, \mathrm{de}^{* * 8}}}$ & $\frac{0,11 \pm}{0,0 \mathrm{~d}^{\mathrm{d} *}}$ & $\frac{330,36 \pm}{19^{* * 3, a b d e^{* * *}}}$ & $\frac{0,84 \pm}{0,01^{\mathrm{d}^{*}}}$ & $\frac{0,20 \pm}{0,00^{c^{*}, b^{* *}}}$ & $\frac{2,04 \pm}{0,04^{b^{*}, c^{8 *}}}$ \\
\hline Grupo & ICG1 & ICG2 & ICR & ICC & ICOI & ICO2 \\
\hline PSI & $\begin{array}{l}116,88 \pm \\
2,02 \mathrm{~d}^{*}\end{array}$ & $\begin{array}{l}198,28 \pm \\
3,43 \mathrm{~d}^{*}\end{array}$ & $\begin{array}{l}99,65 \pm \\
0,97 \mathrm{f}^{\mathrm{f**}}\end{array}$ & $\begin{array}{l}0,04 \pm \\
0,00 \mathrm{ce}^{* *}, \mathrm{f}^{* * 8}\end{array}$ & $\begin{array}{l}3,04 \pm \\
0,08 b c^{z}, f^{* *}\end{array}$ & $\begin{array}{l}8,22 \pm \\
0,37 \mathrm{de}^{* *}, \mathrm{f}^{* * *}\end{array}$ \\
\hline $\mathrm{BH}$ & $\begin{array}{l}121,81 \pm \\
1,87^{f^{*}, c^{8 *}}\end{array}$ & $\begin{array}{l}206,65 \pm \\
3,188^{f^{*}, c^{8 *}}\end{array}$ & $\begin{array}{l}99,74 \pm \\
0,90 \mathrm{~d}^{*}, \mathrm{f}^{* * *}\end{array}$ & $\begin{array}{l}0,04 \pm \\
0,00 \mathrm{c}^{\mathrm{c} * \mathrm{e}, \mathrm{ef} * 8}\end{array}$ & $\begin{array}{l}3,26 \pm \\
0,07 \mathrm{a}^{*}, \mathrm{e}^{* *}, \mathrm{cc}^{\mathrm{s} * *}\end{array}$ & $\begin{array}{l}8,59 \pm \\
0,34 \mathrm{e}^{*}, \mathrm{f}^{\mathrm{*} * *}\end{array}$ \\
\hline QM & $\begin{array}{l}111,83 \pm \\
2,22 \text { bde }^{* *}\end{array}$ & $\begin{array}{l}189,72 \pm \\
3,77 \mathrm{e}^{* *}, \mathrm{bd} \mathrm{d}^{* 8 *}\end{array}$ & $\begin{array}{l}98,55 \pm \\
1,07^{* * *}\end{array}$ & $\begin{array}{l}0,04 \pm \\
0,00 \mathrm{abff}^{* * * 8}\end{array}$ & $\begin{array}{l}2,78 \pm \\
0,08 \mathrm{a}^{\mathrm{a}^{*}, \mathrm{~d}^{* *}, \mathrm{~b}^{* * * *}}\end{array}$ & $\begin{array}{l}8,17 \pm \\
0,41 \mathrm{de}^{* *,}, \mathrm{f}^{* *}\end{array}$ \\
\hline $\mathrm{ARB}$ & $\begin{array}{l}122,28 \pm \\
1,77 \mathrm{a}^{*}, \mathrm{fc} * *\end{array}$ & $\begin{array}{l}207,44 \pm \\
3,00^{b^{*}, f^{* *}, c^{* * *}}\end{array}$ & $\begin{array}{l}97,10 \pm \\
0,85^{b^{*}, e^{* *}, f^{* * *}}\end{array}$ & $\begin{array}{l}0,03 \pm \\
0,00 \text { cef }^{* * *}\end{array}$ & $\begin{array}{l}3,18 \pm \\
0,07 \mathrm{e}^{*}, \mathrm{c}^{* *}, \mathrm{f}^{* * 8}\end{array}$ & $\begin{array}{l}9,46 \pm \\
0,32^{\mathrm{ac} c^{* *}, \mathrm{f}^{* * 8}}\end{array}$ \\
\hline $\mathrm{CR}$ & $\begin{array}{l}119,10 \pm \\
1,75 \mathrm{c}^{* *}\end{array}$ & $\begin{array}{l}202,06 \pm \\
2,97 \mathrm{f}^{*} \mathrm{c}^{* *}\end{array}$ & $\begin{array}{l}100,33 \pm \\
1^{b^{*}, e^{* *}, f^{* * *}}\end{array}$ & $\begin{array}{l}0,04 \pm \\
0,00 \mathrm{a}^{\mathrm{a}^{* 8}, \mathrm{bdf} \mathrm{f}^{* * *}}\end{array}$ & $\begin{array}{l}2,98 \pm \\
0,06^{\mathrm{d}^{*}, b \mathrm{ff}^{* *}}\end{array}$ & $\begin{array}{l}9,69 \pm \\
0,322^{b^{*}, a c^{* *}, f^{*} * *}\end{array}$ \\
\hline $\mathrm{PE}$ & $\begin{array}{l}114,44 \pm \\
2,77^{b^{*}, d^{* *}}\end{array}$ & $\begin{array}{l}194,14 \pm \\
4,70^{b^{*}, d^{* *}}\end{array}$ & $\begin{array}{l}108 \pm \\
1,33 \text { abcde*** }\end{array}$ & $\begin{array}{l}0,05 \pm \\
0,00 \mathrm{c}^{* 8,}, \mathrm{abde} \mathrm{e}^{* * *}\end{array}$ & $\begin{array}{l}2,62 \pm \\
0,11^{\mathrm{ae}^{* *}, \mathrm{db} * *}\end{array}$ & $\begin{array}{l}14,17 \pm \\
0,51^{\text {abcde }}{ }^{* * *}\end{array}$ \\
\hline
\end{tabular}

${ }^{*} \mathrm{p}<0,05,{ }^{* *} \mathrm{p}<0,01,{ }^{* * *} \mathrm{p}<0,001$, ns = não-significante. RCG: relação cernelha garupa; IDT: índice dáctilo torácico; P: peso; IC: indice corporal; IT: indice torácico; ICF: indice de conformação; ICG 1 : indice de carga 1 ; ICG 2: índice de carga 2; ICR: indice corporal relativo; ICO 1: índice de compacidade 1; ICO 2: indice de compacidade 2; ICC: indice de carga na canela. PSI: Puro Sangue Inglês; BH: Brasileiro de Hipismo; QM: Quarto de Milha; ARB: Árabe; CR: Crioulo; PE: Petiço. Médias com letras iguais entre linhas, não diferem estatisticamente pelo teste de Tukey.

As contradições verificadas entre os índices se justificam pelo fato de esta análise ser considerada apenas indicativa da habilidade do animal, assim, seu resultado não deve ser tomado em termos absolutos. De acordo com McManus et al. ${ }^{(6)}$, devem ser consideradas as possíveis compensações entre as medidas que levam à melhoria no desempenho dos animais.

As correlações entre os índices (Tabela 4) demonstraram que equinos com pesos corporais mais elevados tendem a suportar maiores cargas sobre o dorso e são aptos à execução de atividade com tração mais pesada. Todavia, a capacidade do membro deslocar a massa corporal apresentou-se negativa, ou seja, diminui na mesma intensidade em que aumenta o peso sobre o dorso e o tipo de tração. Equinos longilíneos apresentaram menor capacidade de suporte de peso sobre o dorso e menor aptidão para tração em relação aos brevilíneos. Equinos mais compridos em relação à altura, apresentaram menor peso corporal, com maior capacidade dos membros para deslocamento de sua massa corporal. 
Tabela 4. Correlação aos pares dos índices de conformação dos equinos

\begin{tabular}{|c|c|c|c|c|c|c|}
\hline & RCG & IDT & $\mathbf{P}$ & IC & IT & ICF \\
\hline IDT & -0.02 & & & & & \\
\hline $\mathrm{P}$ & $0.32 * * *$ & $-0.26^{* * *}$ & & & & \\
\hline IC & $-0.35 * * *$ & $0.46 * * *$ & $-0.40 * * * *$ & & & \\
\hline IT & -0.08 & $0.20^{*}$ & 0.11 & $0.31 * * *$ & & \\
\hline ICF & $0.21 * *$ & $-0.37 * * * *$ & $0.79 * * *$ & $-0.68 * * * *$ & -0.17 & \\
\hline ICG1 & $0.21 * *$ & $-0.37 * * * *$ & $0.79 * * *$ & $-0.68 * * *$ & -0.17 & $0.99 * * *$ \\
\hline ICG2 & $0.21 * *$ & $-0.37 * * * *$ & $0.79 * * *$ & $-0.68 * * * *$ & -0.17 & $0.99 * * *$ \\
\hline ICR & $-0.47 * * * *$ & $0.25 *$ & $-0.42^{* * * *}$ & $0.51 * * *$ & -0.70 & -0.12 \\
\hline ICO1 & $0.27 * *$ & $-0.35 * * *$ & $0.93 * *$ & $-0.60 * * * *$ & -0.05 & $0.96 * *$ \\
\hline $\mathrm{ICO} 2$ & $-0.21^{*}$ & -0.14 & -0.20 & $-0.32 * * * *$ & $-0.29 * * *$ & $0.34 * * *$ \\
\hline \multirow[t]{2}{*}{ ICC } & $-0.27 * *$ & $0.65 * * *$ & $-0.88 * * *$ & $0.49 * * *$ & -0.03 & $-0.72^{* * * *}$ \\
\hline & ICGl & ICG2 & ICR & ICOI & ICO2 & \\
\hline ICG2 & $0.99 * * *$ & & & & & \\
\hline ICR & -0.12 & -0.12 & & & & \\
\hline ICO1 & $0.96^{* * *}$ & $0.96 * * *$ & $-0.27 * * * *$ & & & \\
\hline $\mathrm{ICO} 2$ & $0.34 * * *$ & $0.34 * * *$ & $0.56 * * *$ & 0.10 & & \\
\hline ICC & $-0.72 * * * *$ & $-0.72^{* * * *}$ & $0.51 * * *$ & $-0.84^{* * * *}$ & $0.20 *$ & \\
\hline
\end{tabular}

${ }^{*} \mathrm{p}<0,05,{ }^{* *} \mathrm{p}<0,01,{ }^{* * *} \mathrm{p}<0,001, \mathrm{~ns}=$ não-significante. RCG: relação cernelha garupa; IDT: índice dáctilo torácico; P: peso; IC: índice corporal; IT: índice torácico; ICF: índice de conformação; ICG 1: índice de carga 1; ICG 2: índice de carga 2; ICR: índice corporal relativo; ICO 1: índice de compacidade 1; ICO 2: índice de compacidade 2; ICC: índice de carga na canela.
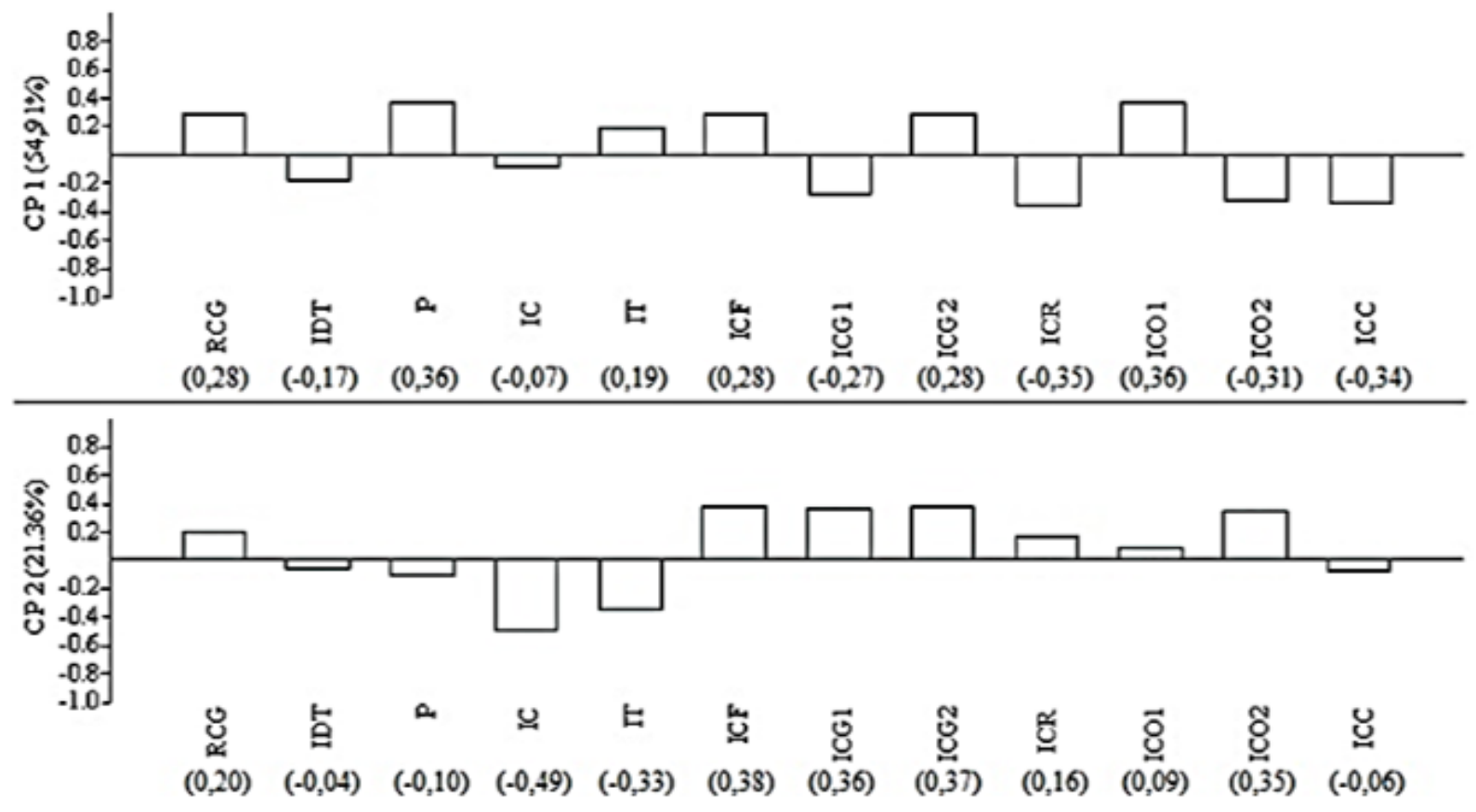

Figura 1. Valores da contribuição das variáveis de acordo com os componentes 1 e 2. PCA1: principal componente 1; PCA2: principal componente 2. RCG: relação cernelha garupa; IDT: índice dáctilotorácico; P: peso; IC: índice corporal; IT: índice torácico; ICF: índice de conformação; ICG 1: índice de carga 1; ICG 2: índice de carga 2; ICR: índice corporal relativo; ICO 1: índice de compacidade 1; ICO 2: índice de compacidade 2 ; ICC: índice de carga na canela. 
Os dois primeiros componentes principais apresentaram percentual de variância acumulada suficiente para justificar 76,27\% da variação (Figura 1). Os índices de P e ICO1 mostraram maior intensidade e correlação positiva com o CP 1. Os RCG, ICF, IT e ICG2 comportaram-se de forma semelhante, mas com menor intensidade. Os demais foram negativamente associados com CP 1, enquanto que os ICG1 e ICG2, ICO2, ICF foram relacionados positivamente com alta intensidade no CP 2.

O CP 1 descreveu animais hipermétricos, altos, com aptidões intermediárias para tração e sela e com equilíbrio entre os membros torácicos e pélvicos. O CP 2 descreveu animais com aptidão para tracionar e suportar peso sobre o dorso mais elevadas, com características de equinos brevilíneos, mais baixos e compridos.

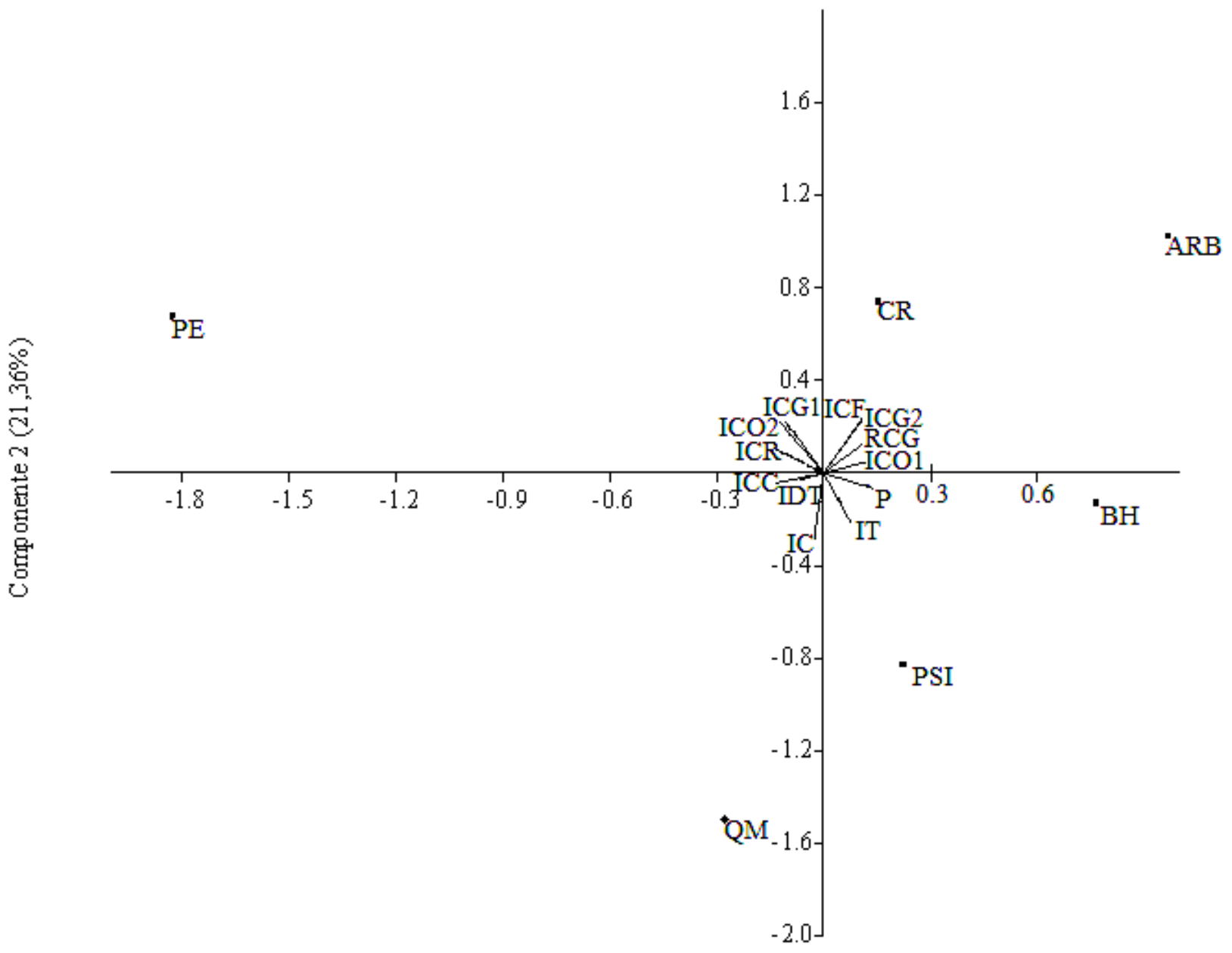

Componente $1(54,91 \%)$

Figura 2. Distribuição dos seis grupos genéticos de equinos em relação aos componentes principais 1 e 2 , obtidos com base nos indices de conformação. ARB: Árabe; PSI: Puro Sangue Inglês; CR: Crioulo; BH: Brasileiro de Hipismo; QM: Quarto de Milha; PE: grupamento genético Petiço. RCG: relação cernelha garupa; IDT: indice dáctilo-torácico; P: peso; IC: índice corporal; IT: índice torácico; ICF: índice de conformação; ICG 1: indice de carga 1; ICG 2: índice de carga 2; ICR: índice corporal relativo; ICO 1: índice de compacidade 1 ; ICO 2: índice de compacidade 2; ICC: índice de carga na canela. 
Analisando-se a distribuição dos seis grupos genéticos em relação aos CP 1 e CP 2 (Figura 2), notou-se que o $\mathrm{PE}$ apresentou menor valor para as características que tiveram maiores valores no $\mathrm{CP} 1$; todavia, para o $\mathrm{CP} 2$, obtiveram-se valores positivos. $\mathrm{O} \mathrm{BH}$ apresentou maiores valores para o $\mathrm{CP} 1$, principalmente próximo ao índice de P e ICO 1. O PSI mostrou perfil longilíneo, com característica de animal de sela, alto e eumétricos. O QM apresentou característica brevilínea e aptidão maior para sela. O ARB apresentou perfil de maior equilíbrio entre seus membros pélvicos e torácicos junto com o $\mathrm{CR}$, e maior capacidade de suporte de peso sobre o dorso, trabalhando ao trote ou a galope.

Categorizaram-se os equinos em cinco grupos, com seis populações distintas (Figura 3). A consistência do padrão de agrupamento foi alta e concordante com o valor do coeficiente de correlação cofenética $(0,87)$.

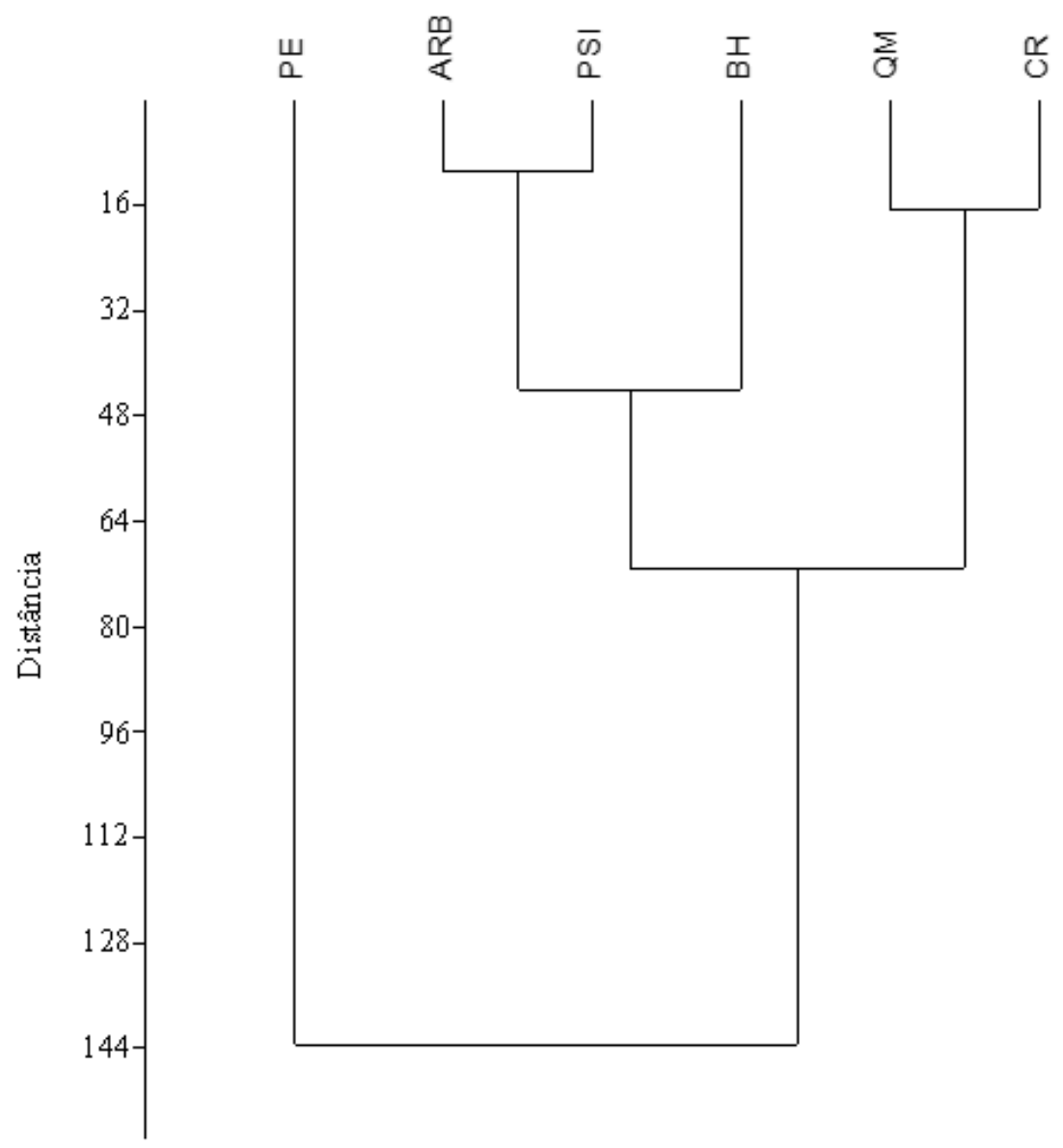

Figura 3. Dendrograma elaborado por algoritmo de grupo emparelhado considerando medida de similaridade euclidiana. Coeficiente de correlação cofenética: 0,87. ARB: Árabe; PSI: Puro Sangue Inglês; CR: Crioulo; BH: Brasileiro de Hipismo; QM: Quarto de Milha; PE: grupamento genético Petiço.

\section{Discussão}

Vários estudos apontam o sexo, bem como o grupo genético, como causas de diferenças entre índices de conformação corporal em equinos ${ }^{(6,8,9,19-21)}$. Todavia, Pimentel et al. ${ }^{(3)}$ verificaram que equinos de 
vaquejada do estado do Rio Grande do Norte demonstraram conformação corporal aproximadamente igual entre os sexos e os grupos. Esse resultado possivelmente se relaciona com a procura em comum dos criadores e competidores de equinos para a modalidade vaquejada, ao contrário dos equinos da presente pesquisa, que são de grupos genéticos com modelo corporal diferente, mantidos em centros equestres distintos com finalidades específicas.

Espera-se que equinos, em relação ao RCG, apresentem uniformidade entre os membros locomotores, pois, segundo McManus et al. ${ }^{(6)}$, quando há desigualdade elevada, pode-se considerar efeito consequente da abertura anormal dos ângulos articulares dos membros torácico e pélvicos, podendo prejudicar tanto o andar como a resistência do equino. No entanto, as correlações indicaram associação de magnitude média e no sentido negativo com o IC, indicando que animais longilíneos, possuem superioridade dos membros pélvicos em relação aos torácicos. O mesmo comportamento pode ser discutido quando se busca um equino brevilíneo para o qual se deseja força (potência), considerando que, nestes casos, o desejável seria o contrário, ou seja, superioridade do membro torácico em relação ao pélvico. Os QM e CR, de acordo com a plotagem dos componentes principais, apresentaram maior profundidade torácica, maior robustez, características apropriadas a equinos dos quais se exige maior força (potência) (brevilíneos).

O maior valor observado para o IC no PSI justifica-se principalmente pelo objetivo de selecionar animais de estatura mais elevada e troncos delgados, pois, morfofuncionalmente, equinos de corrida apresentam tendência a maior comprimento corporal e altura em relação à profundidade torácica ${ }^{(22)}$. Vale ressaltar que a análise de componentes principais plotou o PSI como um grupo com perfil mediolíneo a longilíneo, com característica de animal de sela, altura elevada e peso corporal condizentes com o tipo eumétrico.

A correlação de média magnitude no sentido negativo entre ICR e RCG indicou animais com maior valor de RCG, maior capacidade para atividades que exijam força, pois valor de ICR igual a 100 em equinos indica que o animal possui comprimento corporal proporcionalmente igual à sua altura (intermediário para uso em sela e tração); valores acima de 100 indicam animais com comprimento corporal proporcionalmente superior à sua altura (tração), valores abaixo indicam animais com comprimento corporal proporcionalmente inferior à sua altura (sela).

Essa hipótese é reforçada pela correlação entre IT e ICR $(-0,70)$, em que animais que possuem membros torácicos mais robustos e com boa profundidade torácica estão caracterizados como brevilíneos, enquanto que animais delgados são longilíneos. O ICR foi maior no PE, o que se justifica uma vez que se trata da uniformidade entre o comprimento do corpo e altura de cernelha, sendo esse grupo genético com distribuição menos proporcional pela sua baixa estatura.

Os ICG, ICF, ICO e P indicaram que animais com maior estrutura corporal, maiores perímetros torácicos e dos membros locomotores e, proporcionalmente, menores comprimentos de troncos em relação à altura, possuem superioridade na capacidade de suporte de peso sobre o dorso tanto caminhando como a trote, demonstrando maior aptidão para tração. Vale ressaltar que ICG, ICF e ICO apresentaram correlação negativa com o ICC, indicando que, para selecionar equinos com aptidões para cargas mais elevadas e tração, deve-se buscar indivíduos com maior perímetro de membros locomotores.

A categorização de eumétricos de todos os grupos genéticos considerando o IDT se justifica pela equação do índice, na qual se buscou associar a massa corporal aos membros locomotores (sustentação) e, como todos os animais eram adultos, observou-se maior proporção do perímetro torácico em relação ao perímetro da canela.

A diferenciação do P, com oscilações desde 330,36 kg encontrados em PE até 527,00 kg nos equinos $\mathrm{BH}$, eram esperadas, pois os seis grupos genéticos possuem perfis morfológicos (altura, largura e comprimento) diferentes. A maior capacidade de suporte de peso sobre a canela (ICC) do PE também se justifica pela sua baixa estatura e, quando se plotaram os grupos genéticos sobre os eixos do CP 1 e CP 2, verificou-se que o PE é um animal com menor peso e estatura corporal. Observou-se que o IDT apresentou distância próxima a zero para o componente 1 (CP 1) e 2 (CP 2), sugerindo, portanto, 
a não utilização desse índice na diferenciação entre os grupos.

De acordo com Oom e Ferreira ${ }^{(14)}$, quando os valores para o ICC são muito baixos, estes retratam animais com membros fracos, o que pode comprometer seu desempenho, comprometendo a aptidão motriz para qualquer utilização. Cabral et al. ${ }^{(5)}$ verificaram que animais jovens da raça Mangalarga Machador apresentaram maiores pressões devido ao rápido ganho de peso, sendo essa característica dispersada com o avanço da idade do animal. Já os demais grupos apresentaram melhor distribuição por todo o membro locomotor, tendo em vista que esses são mais proporcionais a toda a estrutura corporal.

O dendrograma reportou a distância menor entre o QM e CR, sendo que ambos possuem porte corporal (peso e altura) intermediário aos seis grupos analisados, possuindo dupla aptidão para execução de tarefas com sela até tração média. O dendrograma apresentou-se consistente, visto que a literatura reporta que dendrogramas com correlação cofenética maior que 0,70 indicam adequação do método de agrupamento para resumir a informação do conjunto de dados ${ }^{(18)}$.

Os grupos PSI, ARB e BH formaram o terceiro grupo, enquanto que o PE apresentou maior distância dos demais em relação à conformação corporal. Esse resultado apresenta consonância com o estudo de Rezende et al. ${ }^{(4)}$, que verificaram que PE apresenta valores para altura de cernelha, comprimento corporal e perímetro torácico inferior quando se compara com o padrão racial mínimo para o registro do $\mathrm{BH}, \mathrm{QM}, \mathrm{CR}, \mathrm{ARB}$ e CR.

\section{Conclusões}

A conformação corporal dos equinos variou conforme o grupo genético e sexo, com maior distância de dissimilaridade do PE em relação aos demais. No geral, todos os grupos apresentaram índice corporal para dupla aptidão. A análise multivariada foi eficiente em agrupar os grupos genéticos dos equinos mais similares para as características de conformação corporal, o que pode auxiliar ou orientar a seleção de características morfofuncionais dos grupos genéticos aqui estudados, em especial, quando empregada em número maior de indivíduos.

\section{Referências}

1. Barker JSF, Moore SS, Hetzel DJS, Evans D, Tan SG, Byrne K. Genetic diversity of Asian water buffalo (Bubalus bubalis): microsatellite variation and a comparison with protein-coding loci. Animal Genetics. 1997;28:(2):103-115.

2. Kane AJ, Stover SM, Gardner TA. Horseshoe characteristics as possible risk factors for fatal musculoskeletal injury of thoroughbred racehorses. American Journal of Veterinary Research. 1996;57:(8):1147-1152.

3. Pimentel MML, Camara FV, Dantas RA, Freitas YBN, Dias RVC, Souza MV. Biometrics of "vaquejada" horses in Rio Grande do Norte, Brazil. Acta Veterinaria Brasilica. 2011;5:(4):376-379.

4. Rezende MPG, Souza JC, Mota MF, Jardim, RJD, Ramires GG, Silva RM, Souza CF. Morfometria corporal de equinos utilizados em trabalho, esporte e lazer em três municípios do Mato Grosso do Sul. Veterinária e Zootecnia. 2014;21:(4):569-583.

5. Cabral GC, Almeida FQ, Quirino CR, Azevedo PCN, Pinto LFB, Santos EM. Morphometric Evaluation of Mangalarga Marchador Horse: Conformation Index and Body Proportions. Revista Brasileira de Zootecnia. 2004; 33:(6):1798-1805.

6. McManus CM, Falcão RA, Spritze A, Costa D, Louvadini H, Dias LT, Teixeira RA, Rezende MJM, Garcia JAG. Caracterização morfológica de equinos da raça Campeiro. Revista Brasileira de Zootecnia. 2005;34:(5):1553-1562. 
7. Parés CasaNova MP. Valoración morfológica de los animales domésticos - Zoometría. In: Sociedad Española de Zooetnólogos. 1nd ed. Madri: Ministerio de Medio Ambiente y Medio Ruraly Marino; 2009. p. 171-198. Disponível em: http://www.magrama.gob.es/es/ganaderia/temas/zootecnia/razas-ganaderas/ publicaciones-interes/LIBRO valoracion_morfologica_SEZ tcm7-306042.pdf. Acesso em setembro de 2011.

8. Rezende MPG, Abreu UGP, Souza JC, Santos AS, Ramires GG, Sitorsky LG. Body morphology of purebred and crossbred Quarter horses used in Lasso competitions in Mato Grosso do Sul. Archivos de Zootecnia. 2015;64:(246):183-186.

9. Rezende MPG, Ramires GG, Souza JCS. Equinos utilizados para tração de carroças em Aquidauana (MS) estão aptos para tal finalidade?. Revista Agrarian. 2013;6:(22):505-513.

10. Hagger C, Hoffer A. Phenotypic and genetic relationships between wither height, heart girth and milk yield in the Swiss Braunvieh and Simmental breeds. Livestock Production Science. 1991;28:(3):265-71.

11. Martin-Rosset W. Particularites de lacroissance et du development ducheval. Revue bibliographique. Annales De Zootechnie. 1983;32:(1):373-380.

12. Franci O, Giogetti A, Gremoli G. Evoluzi one delle caractteristic hemorphologi quenel cavalo avelignese in accrescimento. Zootecnia Nutrizione Animale. 1989;15:(1):373-380.

13. Souza JCS, Rezende MPG, Ramires GG, Gonçalves VT, Souza CF, Oliveira NM, Ribeiro RV. Phenotypic traits of equines raised in the Pantanal of Mato Grosso do Sul. Semina: Ciências Agrárias. 2015;36:(5): 33413352 .

14. Oom MM, Ferreira JC. Estudo biométrico do cavalo Alter. Revista Portuguesa de Ciências Veterinárias. 1987;83:(482):101-148.

15. Peña F, Gómez MD, Bartolomé E, Valera M. Valoración morfológica de los animales domésticos Zoometría, In: Sociedad Española de Zooetnólogos. 1nd ed. Madri: Ministerio de Medio Ambiente y Medio Ruraly Marino; 2009. p. 203-230. Disponível em: http://www.magrama.gob.es/es/ganaderia/temas/zootecnia/ razas-ganaderas/publicaciones-interes/LIBRO_valoracion_morfologica_SEZ tcm7-306042.pdf. Acesso em setembro de 2011.

16. Jolliffe IT. Discarding variables in a principal component analysis. II. Real data. Applied Statistics. 1973;22:(1):21-31.

17. Skoal RA, Rohlf FJ. The comparison of dendograms by objective methods. Taxonomy. 1962;11:(1):33-40.

18. Rohl FJ. Adaptative hierarquical clustering schemes. Systematic Zoology. 1970;19:(1):58-82.

19. McManus CM, Santos AS, Silva JÁ, Louvadini H, Abreu UGP, Sereno JRB, Mariante AS. Body indices for the Pantaneiro horse. Brazilian Journal of Veterinary Research and Animal Science. 2008;45:(5):362-370.

20. Barrey E, Desliens F, Poirel D, Biau S, Lemaire S, Rivero JLL, Langlois B. Early evaluation of dressage ability in different breeds. Equine Veterinary Journal. 2002;34:(1):319-324.

21.Ramos TNM, Cardoso D, Oliveira JV, Bonfim CAM. Caracteristica zoométrica de equinos de raças definidas e não definidas criadas na região de Araçatuda, SP. Boletim da Industria Animal. 2014;71:(3):234-240.

22. Pimentel MML, Pinheiro M, Maria Filho H, Sakamoto SM, Nobre FV, Dias RVC. Biometric parameters of asinines (Equus asinus) used in evidence of race in the state of Rio Grande do Norte. Acta Veterinaria Brasilica. 2014;8:(2):136-143. 